\title{
Frecuencia de caries en niños de 1 a 5 años y conocimientos, actitudes y prácticas de acuerdo a investigaciones realizadas en universidades de Asunción reconocidas por el Ministerio de Educación y Cultura durante los años 2000 al 2013
}

\section{Resumen}

Objetivo: Conocer la frecuencia de caries en niños de 1 a 5 años y conocimientos, actitudes y prácticas de acuerdo a investigaciones realizadas en universidades de Asunción reconocidas por el Ministerio de Educación y Cultura durante los años 2000 al 2013, teniendo en cuenta los criterios de inclusión y exclusión establecidos para la realización del estudio; el $59,2 \%$ corresponden a universidades que se encuentran en gran Asunción, mientras que el $47,1 \%$ corresponden al interior del país. Se identificó un total de 29 investigaciones en niños de 1 a 5 años entre los años 2000 al 2013 referentes a caries y conocimiento, actitud y práctica; siendo más estudiada la variable caries. Los valores del ceod pudieron demostrar un inicio de caries a los 3 años 1.76 aumentando con la edad y llegando a los 5 años a 4.73. La frecuencia de caries se encontró a partir de la edad de 2 años con valores entre 1,64\% a 18,8\%; en la edad de 3 años entre 8 a $40 \%$; a los 4 años entre $15,5 \%$ a $72,7 \%$ y a los 5 años entre $38,7 \%$ a $58 \% ; 50 \%$ de las investigaciones mostraron un nivel de conocimiento y práctica desfavorable.

Palabras claves: Niños de 1 a 5 años, Caries, ceo-d, Conocimientos y prácticas en salud bucal.

Artigo Original

\section{Frequência de cárie em crianças de 1 a 5 anos e conhecimento, atitudes e práticas de acordo com pesquisas realizadas nas universidades reconhecidas pelo Ministério da Educação e Cultura Assunção}

\section{Resumo}

Objetivo: determinar a frequiência de cárie em crianças de 1 a 5 anos e conhecimento, atitudes e práticas, de acordo com pesquisa realizada nas universidades de Assunção reconhecidos pelo Ministério da Educação e Cultura, durante os anos de 2000-2013, considerando os critérios de

\footnotetext{
${ }^{1}$ Egresada de la Facultad de Odontología Universidad Autónoma del Paraguay. (UAP)

${ }^{2}$ Especialista y Máster en Odontopediatría de la Facultad de Odontología Universidad Autónoma del Paraguay. (UAP), Lic. en Psicología especializada en Psicología Clínica y de la Salud, Docente e investigadora con énfasis en epidemiologia
} 
inclusão e exclusão estabelecidos para o estudo. $59,2 \%$ são universidades que estão em grande Asunción, enquanto $47,1 \%$ estão no interior. Foi identificado um total de 29 pesquisas em crianças de 1 a 5 anos entre 2000 e 2013 referentes a cárie e conhecimento, atitudes ou práticas, sendo o componente cariado a variável mais estudada. Os valores de ceo-d médio foram de 1,76 aos 3 anos, aumentando com a idadee chegando a 4,73 aos 5 anos. A freqüência de cárie mostrou, aos 2 anos de idade, valores entre $1,64 \%$ a $8 \%$; aos três anos, ficou entre 18-40\%; aos quatro anos entre $15,5 \%$ e $72,7 \%$ e, aos cinco anos, de $38,7 \%$ para $58 \%$. $50 \%$ das pesquisas mostraram um grau de conhecimento e prática desfavorável.

Palavras-chave: Crianças de 1 a 5 anos, cárie dentária, ceo-d, Saberes e práticas em saúde bucal.

Review Article

\section{Frequency of caries in 1 to 5 year-old children, and knowledge, attitudes and practices according to research conducted in Universities recognized by the Ministry of Education and Culture in Asuncion}

\begin{abstract}
Objectives: To determine the the frequency of caries in children 1 to 5 years-old, and knowledge, attitudes and practices according to research conducted in universities recognized by the Ministry of Education and Culture of Asuncion during years 2000 the 2013, considering the criteria of established inclusion and exclusion for the accomplishment of the study, 59.2\% correspond the great Asuncion, where $47.1 \%$ correspond to the rural area. A total of 29 investigations in children of 1 to 5 years between years 2000 to the 2013 referring ones to caries and knowledge, attitudes and practices were identified; caries being the most studied variable. The values of the $\mathrm{dmf}$ demonstrate caries at age 3 years $(1,76)$ increasing at age 5 years (4.73). Caries frequency was initiated at the age of 2 years with values between 1.64\% to $8 \%$; in the age of 3 years between 18 to $40 \%$; to the 4 years between $15.5 \%$ to $72.7 \%$ and to
\end{abstract}

the 5 years between $38.7 \%$ to $58 \%$; where other $50 \%$ of the investigations showed a level of knowledge and bad practice.

Keys words: Children from 1 to 5 years, Teeth cavity, dmf, Knowledge and practice on dental health.

\section{Introducción}

La caries de la infancia temprana (CTI) se refiere netamente a la enfermedad específica que afecta a la dentición primaria en niños pequeños, cuyos períodos de mayor susceptibilidad se establecen entre los 3 y 4 años e incluye la caries dental del biberón, los niños que sufren este tipo de caries tienden a mantener un riesgo alto para sufrir caries no sólo en dientes deciduos, sino también en la dentición permanente, lo que compromete la salud general de los mismos. Hoy en día, es claro decir que la salud oral no 
es sólo problema del odontólogo; la familia y la comunidad juegan también un papel importante en la promoción de la salud bucal y la prevención de enfermedades. La caries dental se ha considerado como la enfermedad de mayor peso en la historia de la morbilidad bucal a nivel mundial ${ }^{1,2}$.

Si bien la etiología de la CTI es multifactorial se ha establecido que el Streptococcus mutans, inmerso en un biofilm bacteriano, es su principal agente causal, efecto potenciado por características en la dieta de los pacientes infantiles. Los hidratos de carbono fermentables presentes en la dieta son metabolizados por las bacterias produciendo una alteración en la homeostasis bacteriana al disminuir el pH mediante la producción de ácidos y la consecuente desmineralización de los tejidos dentales. También se le debe asociar a factores culturales, socio-económicos y conductuales que juegan un poderoso papel en el desarrollo y progresión de esta enfermedad ${ }^{3}$.

La CTI está estrechamente relacionada con los factores de riesgo, entre los que podemos citar: la dieta, las bacterias intraorales, la composición de la saliva, la capacidad de remineralización de los dientes, basta con que estos factores interactúen para que la enfermedad se establezca en el niño, según la OMS afecta entre el 60\% y $90 \%$ de la población escolar. En relación a los aspectos epidemiológicos, la CTI constituye un serio problema de salud pública. A consecuencia de este daño oral, la CTI en los niños puede causar dolor, dificultades funcionales, desórdenes de salud general, problemas psicológicos, hospitalizaciones y atenciones de urgencia, lo que conlleva a una menor calidad de vida ${ }^{3}$.

La caries dental se puede transmitir y es importante resaltar y conocer que los dientes temporales tienen un menor tiempo eruptivo que los dientes permanentes ${ }^{4}$.

En investigaciones realizadas en el Paraguay hasta el año 2012 sobre prevalencia de caries en niños, pudimos observar que Jacquett Toledo N, Chirife M en el año 2000 en la Pierre Fauchard, demostraron un ceo de 5,6 en niños de 5 años. Alviso Storrer, Chirife, Velásquez en el año 2005 en la Pierre Fauchard, hallaron en niños de 1 a 3 años un ceo de 2.12 y una frecuencia de caries del 41\%. Noguera Riveros, Jacquett Toledo en el año 2011 en la Pierre Fauchard, observaron que la prevalencia de caries en el género femenino es de $12,2 \%$ con un ceo de 2,43. La prevalencia de caries en el género masculino es de $12,4 \%$ y el ceo de 2,47. El ceo en las edades es el siguiente 2 años ( F:0,0 M:0,0 ); 3 años ( F:1,81 M:1,72 ); 4 años (F:1,47 M:1,63 ); 5 años ( F:3,71 M:4,03). Paniagua Cocían, Ayala Haedo, Sotomayor en el año 2012 en la Pierre Fauchard, demostraron una prevalencia de caries en una guardería privada del $22 \%$ y en una guardería pública del $40 \%$ (5-8).

En otras investigaciones Battilana, Fernández en el año 2010 en la UNA, indicaron que solo el 3\% de los niños estudiados no presentaron ningún par de molares homólogos afectados, mientras que en el $96 \%$ se constató por lo menos un par de molares homólogos afectados. Mora Meilicke, Naville Acosta en el año2010 en la UNA, pudieron constatar que el 35,5\% de los pacientes presentó lesión cariosa y el 1,1\% presentaba indicación para endodoncia. Jara Pintos y col en el año 2012 en la UNA, constataron que en la dentición temporaria hubo un total de extracciones dentarias del $28 \%$ y un total de caries del 11,8\%. El 64,4\% de los participantes acudieron por motivos de dolor y solo el 35,5 \% para control. Báez Maidana, Gavilán Mareco, Núñez Mendieta hallaron que las causas principales de las extracciones pre- 
maturas fueron la gangrena $64,6 \%$, caries profunda $23,7 \%$, y la pulpitis en un $1,4 \%$ (9-12).

Los datos anteriormente presentados evidencian la presencia de caries en niños en edades tempranas por lo que el objetivo de este trabajo es Conocer la frecuencia de caries en niños de 1 a 5 años y CAP (conocimientos, actitudes y prácticas) de acuerdo a investigaciones realizadas en universidades de Asunción reconocidas por el MEC (Ministerio de Educación y Cultura) durante los años 2000 al 2013, los datos encontrados podrían ser de relevante valor para la evaluación de la posibilidad de cambios futuros en el modo de planificación y ejecución de los programas de salud en nuestro país.

\section{Objetivo General}

Conocer la frecuencia de caries en niños de 1 a 5 años y CAP (conocimientos, actitudes y prácticas) de acuerdo a investigaciones realizadas en universidades de Asunción reconocidas por el MEC(Ministerio de Educación y Cultura) durante los años 2000 al 2013.

\section{Material y método}

Para llevar a cabo la lectura crítica de la literatura fueron localizados 29 investigaciones originales a través de datos recolectados en las diferentes Universidades de Odontología reconocidas por el MEC (Ministerio de Educación y Cultura) públicas y privadas, la Universidad Nacional de Asunción "UNA", Universidad Católica "Nuestra Señora de la Asunción", Universidad del Norte, Universidad Autónoma de Asunción "UAA", Universidad Privada del Este, Universidad Autónoma del Paraguay "Pierre
Fauchard" "UAP", Universidad Autónoma del Paraguay "UAP", Universidad del Pacífico "UP", Universidad Politécnica y Artística, Universidad Metropolitana de Asunción, Universidad Internacional Tres Fronteras, Universidad Central del Paraguay, Universidad Nacional de Concepción, Universidad San Lorenzo, Universidad Autónoma del Sur, Universidad Hispano Guaraní para el desarrollo humano, Universidad Nacional de Caaguazú, donde cumpliendo el objetivo del trabajo se visitaron las universidades de Asunción, tales como la Universidad Autónoma del Paraguay "Pierre Fauchard" "UAP", Universidad del Pacífico "UP", la Universidad Nacional de Asunción "UNA", Universidad del Norte, Universidad Autónoma de Asunción "UAA", Universidad Politécnica y Artística, Universidad Metropolitana de Asunción, Universidad Hispano Guaraní para el desarrollo humano; previamente a la revisión de las investigaciones en dichas bibliotecas se procedió a la entrega de una nota de permiso al Rector y/o Director Académico de cada universidad con el fin de que se autorice el acceso a dichas investigaciones; de estas universidades fueron utilizadas 29 investigaciones originales depregradorealizadas por los alumnos de la carrera de odontología de la Universidad Autónoma del Paraguay "Pierre Fauchard", la Universidad Nacional de Asunción y la Universidad Autónoma de Asunción "UAA". Fueron incluidos en la revisión aquellos artículos que cumplieron con los siguientes criterios: eran artículos científicos originales, tesis no publicadas. Poseían medición de la incidencia, prevalencia o frecuencia de la caries dental asociada a factores de riesgo de la enfermedad, eran publicaciones de los años 2000 al 2013 y por último, la edad de los niños incluidos en la muestra estaba comprendida entre los 1 y 5 años, incluyendo niños con 
características diferentes y CAP realizados en madres/padres, tutores y profesores, también se obtuvieron datos sobre el nivel de consultas odontológicas, pediátricas y ginecológicas en el MSPBS. Se excluyeron las universidades en las que no fueron firmadas las notas de permiso entregadas al Rector y/o Director Académico como la Universidad Central del Paraguay, y también las universidades que no abrieron la carrera de odontológica en el año 2013 como la Universidad Metropolitana de Asunción.

Los valores mencionados de los rangos de ceo-d y frecuencia de caries concluidos en el trabajo se calcularon sumando los valores finales encontrados en las investigaciones, dividiendo el número de investigaciones; es decir se buscó presentar los promedios de acuerdo a las investigaciones encontradas.

Una vez obtenido el número de artículos previstos, se llevaron a cabo los siguientes pasos: elaboración de fichas, cuantificación de los valores del ceo, frecuencia de caries y datos sobre CAP, para el análisis de dichos datos se utilizó un paquete estadístico tipo Excel, utilizando estadística descriptiva.

Tabla 1. Universidades de odontología del Paraguay (Asunción e interior del país) reconocidas por el MEC. (n: 17).

\begin{tabular}{|c|c|c|}
\hline Universidades & Cantidad & Porcentaje \\
\hline Asunción & 9 & $52.9 \%$ \\
\hline $\begin{array}{c}\text { Interior } \\
\text { del país }\end{array}$ & 8 & $47.1 \%$ \\
\hline TOTAL & 17 & $100 \%$ \\
\hline
\end{tabular}

Todas las investigaciones revisadas fueron de diseño observacional descriptivo de corte trasverso y las inspecciones bucales los autores lo realizaron bajo luz natural y con instrumentos de inspección habitual (espejo bucal sin aumento) previa realización de la higiene bucal a los niños.

\section{Resultados}

En cuanto al número de universidades de odontología del Paraguay (públicas y privadas), se encontró que existe un total de 17 universidades de odontología, tanto en el gran Asunción como en el interior del país (tabla 1).

En cuanto a las universidades de odontología del Paraguay divididas por departamentos, se observó que el 52,9\% corresponde al departamento central el 17,6\% a Ciudad del Este, $11,8 \%$ a Villa Rica, 5,9\% a Concepción, el $5,9 \%$ a Coronel Oviedo y el 5,9\% a Caaguazú (tabla 2).

Tabla 2. Universidades de odontología del Paraguay (divididas por departamentos) reconocidas por el MEC. (n: 17).

\begin{tabular}{|c|c|c|}
\hline Departamento & Universidades & Porcentaje \\
\hline Central & 9 & $52.9 \%$ \\
\hline Ciudad del Este & 3 & $17.6 \%$ \\
\hline Villa Rica & 2 & $11.8 \%$ \\
\hline Concepción & 1 & $5.9 \%$ \\
\hline Coronel Oviedo & 1 & $5.9 \%$ \\
\hline Caaguazú & 1 & $5.9 \%$ \\
\hline
\end{tabular}


El total de las investigaciones halladas sobre frecuencia de caries, CAP y frecuencia de caries en niños con características especiales en las distintas universidades; corresponde al $65,5 \%$ en la Universidad Autónoma del Paraguay "Pierre Fauchard" "UAP", el 31,0\% en la Universidad Nacional de Asunción "UNA" y el 3,4\% en la Universidad Autónoma de Asunción "UAA" (tabla 3 y figura 1).

En la Universidad Autónoma del Paraguay "Pierre Fauchard" "UAP" y en la Universidad Nacional de Asunción "UNA" se encontró 48,3\% de caries (tabla 4).

En cuanto al Análisis de las investigaciones referente a caries encontradas en la Universidad Autónoma del Paraguay "Pierre Fauchard" "UAP"(n: 19), se observó el autor, año, lugar, edad, la población y los resultados referentes al ceo y a la frecuencia de caries. En cuanto a la frecuencia de dientes cariados se encontró valores a partir de los 2 años con una frecuencia que variaron entre 1,64 al 18,8\%, así también a los 3 años entre $8 \%$ a $40 \%$, a los 4 años entre $15,5 \%$ a $72,5 \%$ y a los 5 años entre $38,7 \%$ a $58 \%$.

En cuanto a los valores promedio poblacional del ceo-d teniendo en cuenta el número de investigaciones encontradas en niños de 2 a 5 años en trabajos realizados en la Universidad Autónoma del Paraguay "Pierre Fauchard" "UAP", se observó que los valores aumentaron según la edad, encontrando un ceo-d de 1,76 en niños de 3 años; 1,56 corresponde a niños de 4 años y 4,73 en niños de 5 años. (tabla 5).

En cuanto a los datos sobre el nivel de consultas odontológicas, ginecológicas y pediátricas en el MSPBS encontradas en la Universidad Autónoma del Paraguay "Pierre Fauchard

\begin{tabular}{|c|c|c|}
\hline Universidad & Investigaciones & Porcentaje \\
\hline UNA & 9 & $31 \%$ \\
\hline Univ. del Norte & 0 & $0 \%$ \\
\hline UAA & 1 & $3.4 \%$ \\
\hline UAP & 19 & $65.5 \%$ \\
\hline UP & 0 & $0 \%$ \\
\hline $\begin{array}{c}\text { Univ. Politécnica } \\
\text { y Artística }\end{array}$ & 0 & $100 \%$ \\
\hline $\begin{array}{c}\text { Univ. } \\
\text { detropolitana Asunción }\end{array}$ & $20 \%$ \\
\hline $\begin{array}{c}\text { Universidad } \\
\text { Hispano } \\
\text { Guaraní }\end{array}$ & 0 & $0 \%$ \\
\hline $\begin{array}{c}\text { TOTAL } \\
\text { Und }\end{array}$ & 0 & \\
\hline
\end{tabular}

Tabla 3. Número de investigaciones halladas sobre frecuencia de caries, CAP y frecuencia de caries en niños con características especiales en las diferentes universidades de Asunción reconocidas por el MEC. (n: 29).

"UAP" entre el año 2007 y 2010 fueron consultas odontológicas: 4,96\%-7,33\% en comparación a las consultas pediátricas de 95,04\% - 92,67\%, y las consultas odontológicas: 5,82\% - 7,28\% comparando con las ginecológicas 94,18\% $92,72 \%$.

En cuanto a las investigaciones encontradas sobre CAP (n: 9) en la Universidad Autónoma del Paraguay "Pierre Fauchard" "UAP", los resultados oscilaron entre conocimiento de la 
salud bucodental regulary practica desfavorable, en lo relacionado a la salud bucal de sus hijos se encontró conocimiento y actitud mala.En cuanto a los datos encontrados sobre caries en la Universidad Nacional de Asunción "UNA" (n:3), se encontró una frecuencia de caries del $35,5 \%$ y nivel de conocimiento bueno entre $56 \%$ y $77 \%$.

\section{Discusión}

En el presente estudio que lleva como tema Frecuencia de caries en niños de 1 a 5 años y CAP de acuerdo a investigaciones realizadas en

Tabla 4. Investigaciones halladas sobre frecuencia de caries, $C A P$, frecuencia de caries en niños con características especiales y datos sobre el nivel de consultas del MSPBS en la Universidad Autónoma del Paraguay "Pierre Fauchard" "UAP", Universidad Nacional de Asunción "UNA" y en la Universidad Autónoma de Asunción "UAA". (n: 29).

\begin{tabular}{|c|c|c|}
\hline & Investigaciones & Porcentaje \\
\hline Caries & 14 & $\% 48,3$ \\
\hline Caries y CAP & 2 & $\% 6,9$ \\
\hline CAP & 8 & $\% 27,6$ \\
\hline $\begin{array}{c}\text { Niños con } \\
\text { capacidades } \\
\text { diferentes }\end{array}$ & 3 & $\% 10,3$ \\
\hline $\begin{array}{c}\text { Datos sobre } \\
\text { el nivel de } \\
\text { consultas } \\
\text { en el MSPBS }\end{array}$ & 2 & $\% 6,9$ \\
\hline \begin{tabular}{c} 
Total: \\
\hline
\end{tabular} & 29 & $\% 100$ \\
\hline
\end{tabular}

universidades de Asunción reconocidas por el Ministerio de Educación y Cultura (MEC) desde el año 2000 al 2013, se puede observar una alta frecuencia de caries temprana en niños y niñas de 1 a 5 años, que va aumentando con la edad.

Zaror Sánchez C, Pineda Toledo P, Orellana Cáceres J. J. 1, en el año 2011 en un estudio realizado en Calbuco Chile, demostraron que el ceo-d en niños de 2 a 4 años corresponde a 3, la prevalencia de caries de la infancia temprana corresponde a $70 \%$, en cuanto a los niños de 2 años las lesiones incipientes de caries corresponde al $28 \%$, obteniéndose un mayor porcentaje en niños de 4 años con un $88 \%$, difiriendo con este trabajo en el cual se encontró que en los niños de 2 años se hallaron valores entre 1,64\% a $18,8 \%$ y en la edad 4 años entre 15,5\% a 72,7\%.

En la investigación que realizaron Gispert Abreu E, Herrera Nordet M, Lazo F', en el año 2006 realizado en el Área "Aballí” Municipio de la Habana Vieja, observaron que en los niños de 3 años se encontró una afectación por caries de $65,3 \%$ que dependió de forma significativa

Tabla 5. Valores de ceo-d en niños de 2 a 5 años en investigaciones realizadas en la Universidad Autónoma del Paraguay "Pierre Fauchard" "UAP". n: 19

\begin{tabular}{|c|c|}
\hline Edad & ceo-d \\
\hline años 2 & 0 \\
\hline años 3 & 1,76 \\
\hline años 4 & 1,56 \\
\hline años 5 & 4,73 \\
\hline
\end{tabular}


del grado de infección por Streptococcus mutans (Sm), estos datos son similares con este trabajo en el cual se pudo demostrar que el inicio de caries de acuerdo con el ceo se inicia a los 3 años.

Cabrera Escobar D y col' ${ }^{1}$, en al año 2009 realizado en Habana Cuba, observaron que la frecuencia de caries en niños de 2 a 4 años corresponde al 15\%, los niños de alto riesgo fueron significativamente los de mayor prevalencia de caries, con $47,8 \%$ de afectados, coincidiendo con este trabajo en el cual se observó que la frecuencia de caries a los 2 años estuvo en el rango de $15,5 \%$.

Un estudio que fue realizado por Díaz Cárdenas S, González Martínez F. 2', en el año 2010 en Cartagena Indias Colombia, observaron que en niños de 4 a 5 años la prevalencia de caries fue del 51\%, difiriendo de este estudio en el cual se demostró que a los 4 años se halló una frecuencia de caries entre $15,5 \%$ a $72,7 \%$ y a los 5 años entre $38,7 \%$ a $58 \%$.

\section{Conclusión}

En el total de universidades reconocidas por el MEC se realizaron un total de 29 investigaciones en niños de 1 a 5 años entre los años 2000 al 2013 referentes a caries y CAP; siendo más estudiada la variable caries. Entre las universidades donde más trabajo se encontró figura en primer lugar la UAP, seguida por la UNA. Los valores del ceo-d pudieron demostrar un inicio de caries a los 3 años 1.76 aumentando con la edad y llegando a los 5 años a 4.73 .

\section{Recomendaciones}

$\mathrm{Al}$ analizar la frecuencia de caries en niños de 1 a 5 años y CAP de acuerdo a investigaciones realizadas en universidades de Asunción reconocidas por el MEC, se recomienda que es fundamental implementar programas de prevención de caries de la infancia temprana, debido a que se encontró en los trabajos revisados que la caries en la infancia temprana comienza a los 2 años, y en el Paraguay los programas de prevención comienzan a los 6 años y a través de estos programas motivar a los padres/madres/tutores a promover el cuidado de la salud bucal de sus niños.

Falta fomentar el trabajo interdisciplinario entre odontólogos, médicos y especialistas pediatras parael cuidado dela salud bucaldel niño/a, debido a la poca concurrencia de los niños/as actualmente en las consultas odontológicas en nuestro país. Así también el trabajo interdisciplinario con las gestantes teniendo en cuenta que la salud bucal de la madre tiene repercusiones en la futura salud bucal del hijo.

Cabe destacar la importancia que tienen las investigaciones a nivel mundial, y por ende se deberían realizar más investigaciones en el Paraguay que posean una correcta metodología, esto es relevante debido a que muchas de las investigaciones encontradas no contaban con datos bien detallados.

\section{Referencias bibliográficas}

1. Cabrera Escobar D y col. Riesgo de caries dental en niños atendidos en el hogar en el período 2006-2007. Revista Cubana Estomatol. Junio 2009 [citado 2013 Jun 05]; 46(2). Disponible en: http://scielo.sld.cu/scielo.php?script=sci_ arttext\&pid=S0034-75072009000200002\&lng=es. 
2. Díaz Cárdenas S, González Martínez F. Prevalencia de caries dental y factores familiares en niños escolares de Cartagena de Indias, Colombia. Revista Salud pública. Octubre 2010 [citado 2013 Jun 05]; 12(5): 843-851. Disponible en: http://www.scielo.org.co/scielo.php?script=sci_arttext\&pid=S0124-00642010000500014\&lng=en.

3. Gispert Abreu E, Herrera Nordet M, Lazo F. Prevención indirecta de caries en la temprana infancia: Área "Aballi”". II Parte. Revista Cubana Estomatol. Diciembre 2006 [citado 2013 Jun 05]; 43(4). Disponible en: http://scielo.sld.cu/scielo. php?script=sci_arttext\&pid=S0034-75072006000400004\&lng=es.

4. Zaror Sánchez C, Pineda Toledo P, Orellana Cáceres J. J. Prevalencia de caries temprana de la infancia y sus factores asociados en niños chilenos de 2 y 4 años. Revista Int. J. Odontostomat. Agosto 2011 [citado 2013 Jun 05]; 5(2): 171-177. Disponible en: http://www.scielo.cl/scielo.php?script=sci_arttext\&pid=S0718-381X2011000200010\&lng=es. http://dx.doi. org/10.4067/S0718-381X2011000200010.

5. Jacquett Toledo N. L, Chirife M. T, Samudio M. Efectividad de un programa preventivo en la reducción del índice de caries en niños de una escuela pública de Asunción. (Tesis doctoral). Universidad Autónoma del Paraguay “Pierre Fauchard". 2000.

6. Alviso Storrer C, Chirife M. T, Velasquez G. Caries de la infancia temprana según determinantes conductuales, nutricionales y asociados a la transmisión de microorganismos. (Tesis). Universidad Autónoma del Paraguay “Pierre Fauchard". 2005.

7. Noguera Riveros A. E, Jacquet Toledo N. L. Prevalencia de la succión no nutritiva e índice de caries en niños de escuelas maternales de la ciudad de San Lorenzo - Año 2011. (Tesis doctoral). Universidad Autónoma del Paraguay “Pierre Fauchard". 2012.

8. Paniagua Cocian D. J, Ayala Haedo C, Sotomayor R. Condición oral y prevención en niños de guarderías en Asunción en el año 2012. (Tesis doctoral). Universidad Autónoma del Paraguay “Pierre Fauchard”. 2012.

9. Barrios Battilana M. C, Fernández Jara L. E. Benítez de Forcadell S. Frecuencia de caries en molares temporarios homólogos en niños que acudieron a la cátedra de odontopediatria de la facultad de odontología de la UNA en el año 2009. (Trabajo de fin de grado). Universidad Nacional de Asunción “UNA”. 2010.

10. Mora Meilicke M. O, Naville Acosta C. N, Maldonado de Aquino C. Frecuencia de perdida de segundos molares temporarios en niños que acudieron al servicio de cirugía pediátrica en la facultad de odontología de la universidad nacional de Asunción en el año 2010. (Trabajo de fin de grado). Universidad Nacional de Asunción “UNA”. 2011. 11. Jara Pintos C. M, Manzur Ayala M. N. y Col, Benítez de Forcadell S, Amarilla Guirland A. Atención odontológica integral aplicada a usuarios que asisten al instituto nacional de protección a personas excepcionales en el año 2012. (Trabajo de investigación presentado como requisito de graduación para optar al título de Doctor en odontología). Universidad Nacional de Asunción “UNA”. 2012.

12. Báez Maidana C, Gavilán Mareco L. C, Caballero Ramírez E, Núñez Mendieta H. Perdida prematura de dientes temporarios en niños que acudieron a la clínica de la catedra de odontología integral del niño y del adolescente de la facultad de odontología de la Universidad Nacional de Asunción. Años 2009 - 2012. (Trabajo de investigación presentado como requisito de graduación para optar al título de Doctor en odontología). Universidad Nacional de Asunción “UNA". 2013. 
13. Cabral Fernández M. M, Jacquett Toledo N. L. Perdida prematura de dientes temporarios en niños de una escuela pública de la ciudad de San Lorenzo. (Tesis doctoral). Universidad Autónoma del Paraguay “Pierre Fauchard”. 2010. 14. Silva Noguera L M, Ayala Haedo C. Prevalencia de caries en infantes en una escuela presbiteriana de la ciudad de Lambaré y una guardería privada de la ciudad de Asunción Año 2011. (Tesis doctoral). Universidad Autónoma del Paraguay "Pierre Fauchard". 2011.

15. Ovelar Pérez M, Ayala Haedo C. Frecuencia de caries de biberón en niños de 4 y 5 años que asisten a la escuela graduada número 300 Maestro Herminio Giménez 2011. (Tesis doctoral). Universidad Autónoma del Paraguay "Pierre Fauchard". 2011.

16. Fariña P. R, Ayala Haedo C. Prevalencia de caries en molares temporarios en niños de 3 a 5 años que acudieron a la catedra de odontopediatria II en el periodo 2011. (Tesis doctoral). Universidad Autónoma del Paraguay “Pierre Fauchard". 2012.

17. Álvarez Caballero L. R. y col. Índice de caries escolares y grado de conocimientos y práctica de los padres en relación a la prevención bucodental de sus hijos en dos escuelas de la ciudad de Santa Rita durante el año lectivo 2011. (Tesis doctoral). Universidad Autónoma del Paraguay “Pierre Fauchard". 2011.

18. Escumbarti Torales M. R, Ayala Haedo C. Estado de salud bucal en niños escolares de 5 a 10 años de edad de una escuela privada de la ciudad de San Lorenzo. (Tesis doctoral). Universidad Autónoma del Paraguay “Pierre Fauchard". 2012.

19. Páez Caballero M. H, Ayala Haedo C. Perdida prematura de dientes temporarios anteriores en niños que concurrieron a la catedra de odontopediatria de la clínica integrada Pierre Fauchard de marzo a noviembre del dos mil diez. (Trabajo de culminación de curso) Universidad Autónoma del Paraguay “Pierre Fauchard”. 2013.

20. Escumbarti de Cabañas J, Ayala Haedo C. Prevalencia de caries de la infancia temprana en niños de maternal, jardín, preescolar y 1er grado de la escuelas básica número 4916 Reino de España y la escuela privada Sagrada Familia de San Lorenzo. (Tesis doctoral). Universidad Autónoma del Paraguay “Pierre Fauchard”. 2012.

21. Silva Casco M, Ayala Haedo C. Evaluación del nivel de educación en salud bucal de los padres de los niños de 5 a 9 años que asisten en las escuelas de la ciudad de José Falcón Chaco - Paraguay. (Tesis doctoral). Universidad Autónoma del Paraguay "Pierre Fauchard". 2011.

22. Osorio Benítez R, Ayala Haedo C. Nivel de conocimientos, actitudes y conductas de las madres sobre la salud bucal de sus hijos durante el año 2008. (Tesis doctoral). Universidad Autónoma del Paraguay “Pierre Fauchard”. 2011.

23. Dagogliano Castellani G, Jacquett Toledo N. L. Conocimiento de los padres sobre la importancia de la dentición temporal y prevención de caries dental en una escuela pública y privada de Asunción. (Tesis doctoral). Universidad Autónoma del Paraguay "Pierre Fauchard". 2011.

24. Benítez Núñez R.M, Becker M. Conocimiento y prácticas sobre riesgos cariogénicos de padres de niños de temprana infancia que acudieron al centro de Salud Santo Domingo, Lambaré, año 2011. (Tesis). Universidad Autónoma del Paraguay "Pierre Fauchard". 2012.

25. Vera Bogado C. G, Jacquett Toledo N. L. Frecuencia de atención de niños que acudieron a las consultas odontológicas y pediátricas en las diferentes regiones sanitarias del Ministerio de Salud Pública y Bienestar Social durante los años 2007 y 2010. (Tesis). Universidad Autónoma del Paraguay “Pierre Fauchard”. 2011.

26. Spiridonoff Benítez T, Jacquett Toledo N. L. Frecuencia de atención odontológica y ginecológica de embarazadas que acudieron a los diferentes servicios de las regiones del Ministerio de Salud Pública y Bienestar Social durante los años 2007 y 2010. (Tesis doctoral). Universidad Autónoma del Paraguay “Pierre Fauchard”. 2010.

27. Bogado López R. A, Ayala Haedo C. Nivel de conocimiento sobre medidas preventivas en salud bucodental en 
docentes de educación escolar básica de las escuelas de la zona céntrica de la ciudad de Capiatá durante el año 2011. (Tesis doctoral). Universidad Autónoma del Paraguay “Pierre Fauchard”. 2011.

28. Centurión C. C, Caldarera A. R, Rodas L. B, Benítez de Forcadell S. Conocimiento y práctica sobre prevención en salud bucodental de gestantes que acudieron al centro Materno Infantil de la Facultad de Ciencias Médicas de la Universidad Nacional de Asunción en el año 2009. (Trabajo de fin de grado). Universidad Nacional de Asunción “UNA”. 2010. 29. Ayala Benítez A. L. y col. Conocimiento y práctica de salud bucodental de gestantes que acudieron a hospitales públicos de Asunción, Encarnación y Ciudad del Este en el año 2012. (Trabajo de fin carrera). Universidad Nacional de Asunción "UNA". 2012

30. Mesquita Carreras M. B, Benítez de Forcadell S. Conocimiento sobre prevención y práctica de salud bucodental de padres de niños con capacidades diferentes que participan del programa educativo para niños con discapacidad física en el año 2010. (Trabajo de fin de grado). Universidad Nacional de Asunción “UNA”. 2010.

31. Espínola Benítez V. M, Ledesma Campos N. P, Benítez de Forcadell S. Condición oral de niños y jóvenes con capacidades diferentes que acuden al servicio odontológico del Instituto Nacional de Protección a personas excepcionales en el año 2010. (Trabajo de fin de grado). Universidad Nacional de Asunción “UNA”. 2010.

32. Espínola Hidalgo. M. M. y col. Manifestaciones bucales en la enfermedad celiaca en pacientes de 4 a 18 años de edad de la Fundación Paraguaya de Celiacos (FUPACEL) en el año 2012. (Trabajo de fin de grado). Universidad Nacional de Asunción “UNA". 2012.

33. Ministerio de Salud Pública y Bienestar Social (Paraguay) y Organización Panamericana de la salud. Encuesta nacional de salud oral 2008. http://www.mspbs.gov.py/bucodental/.

34. Ministerio de Salud Pública y Bienestar Social (Paraguay) y Organización Panamericana de la salud. Evaluación del programa "Salvemos al primer molar" y la estrategia PRAT del Ministerio de Salud Pública y Bienestar Social del Paraguay 2012. http://www.mspbs.gov.py/bucodental/.

35. Ministerio de Salud Pública y Bienestar Social (Paraguay) y Organización Panamericana de la salud. Descripción operativa del programa "Salvemos al primer molar" 2000. http://www.mspbs.gov.py/bucodental/.

36. Pangrazio de Kegler. N. Análisis Situacional de la Odontología en el Paraguay. 2009.

Recibido 20/03/2015

Aceptado 02/07/2015

Correspondencia: Ninfa Jacquet Toledo ninfajacquett@gmail.com 